\title{
Flight of Tektites from Meteorite Impact
}

\author{
Erwin David \\ French-German Research Institute, F 68 St. Louis, Ht. Rhin, France \\ (Z. Naturforschg. 21 a, 1133-1137 [1966] ; received 4 April 1966)
}

Dedicated to Professor Dr. W. Gentner on the occasion of his 60th birthday

\begin{abstract}
In large meteorite impacts, especially of stony meteorites, of the order of magnitude of the Ries event, some $\mathrm{km}^{3}$ of rock are evaporated, whereby a huge blast is yielded. The expansion flow of this blast provides appropriate means for expelling tektites out of the atmosphere in space orbits. Oblique and irregular impact offer possibilities for rock transformed by shock to viscous glass to enter the expansion flow.
\end{abstract}

The fact that tektites had their origin in terrestrial meteorite impacts may be regarded as proven in the cases of Ries and Bosumtwi and as most likely for the American and Australasian tektites. Up to date it is an unsolved problem, how the tektites managed to fly over distances of several hundreds to several thousands of $\mathrm{km}$. On the other hand there exist impact craters of comparable size which do not seem to be connected with tektites. This means, the flight of tektites needs some special conditions which may be met or not.

In order to explain the problematic penetration of the atmosphere it has been proposed, that the tektites should fly as a jet ${ }^{1}$. This jet is formed as Munro or shaped charge jet by implosion of the hole which an impacting iron meteorite leaves behind.

The inner surface of this hole would be stony matter but due to severe shock heating by impact it has been transformed into the gaseous state. The matter, colliding on the axis of formation of the jet will further be heated by this collision and only that matter, which collides first, contributes to the jet. Such a hot gaseous jet cannot yield rather cool, still highly viscous tektites.

This is the significant difference between experimental light gas gun impacts and natural meteorite impacts. The first with an impact velocity of 6 to $8 \mathrm{~km} / \mathrm{s}$ lead chiefly to hydrodynamic flow, the latter with impact velocities from $20 \mathrm{~km} / \mathrm{s}$ on are phenomena explained to a large extent by gas dynamics. In the experimental field they have their only analogon in wire explosions.

\footnotetext{
1 V. VAND, Nature London 209, 496 [1966].
}

The impact event is a somewhat complicated one which is three dimensional in space and time dependent. For a rough estimate the author uses plane and spherical shock waves and hopes by this procedure to arrive at correct qualitative features. Further quantitative improvements of course remain desirable.

\section{Basic elements}

For the shocks in the impacted ground and in the meteorite the shock wave properties of granite ${ }^{2}$ are used as a somewhat reasonable mean value for the stony matters involved. Fig. 1 contains shock velocity $U$ and particle velocity $u$ as function of pressure $p$.

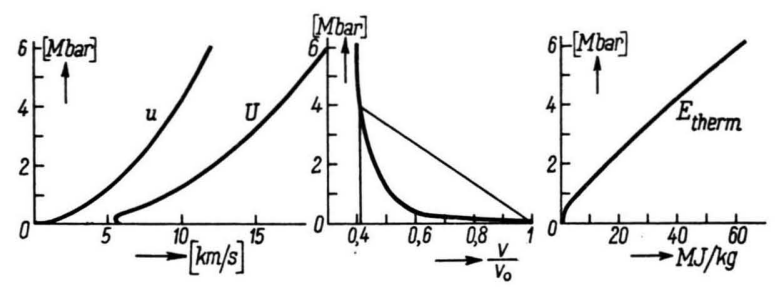

Fig. 1. Shock wave properties for granite.

Departing from measured $U$ and $u$ and the initial specific volume $V_{0}$ the pressure $p$, specific volume $V$ and specific internal energy $E$ can be evaluated by application of the Hugoniot equations. This internal energy $E$ is the area of the triangle, drawn over the graph $V / V_{0}$ for the example $p=4 \mathrm{Mbar}$. If we neglect the difference between the Hugoniot graph

2 D. B. Lombard and F. L. Adelman, The Hugoniot Equation of State of Granite. University of California, Livermore, Rept. UCRL 6419. 
$V / V_{0}$ and the graph of the adiabatic expansion ${ }^{3}$ then the part of the triangle to the left of the graph is the elastic energy of compression, the part to the right is the thermal specific energy $E_{\text {therm }}$ which remains as heat in the shocked matter, when it has expanded to initial volume again. About $1.5 \mathrm{MJ} / \mathrm{kg}$ causes high viscous, $3 \mathrm{MJ} / \mathrm{kg}$ low viscous melting and $8 \mathrm{MJ} / \mathrm{kg}$ total evaporation including the heating to the boiling point. The corresponding shock pressures are 0.5 to $0.8 \mathrm{Mbar}$ for melting and 1.3 Mbar for evaporation.

The graphs shown in Fig. 1 do not deviate severely from those for other rocks, basalt or limestone for instance, and as such can be used in our qualitative treatment for all rocks involved.

The dominating effect for the decrease of a spherically diverging shock is the decrease of particle velocity $u$ with $r^{-3 / 2}$ due to geometric spreading. Minor effects are neglected. The drop of shock wave amplitude with distance is so steep, that distances of interest, as for instance the limits of melting, will never come out noticeably incorrect.

In general it should be emphasized once more that the mechanical part of the phenomenon is the counterplay of inertial forces and pressure gradient or pressure step forces. Rigidity only determines the late stages of the event which we shall not discuss in this paper.

In the following section we investigate the course of events for an example with dimensions which could be similar to that of the Ries event ${ }^{4}$ :

diameter of meteorite: $1.2 \mathrm{~km}$, mass: $2.5 \times 10^{12} \mathrm{~kg}$, velocity (vertical component) : $22 \mathrm{~km} / \mathrm{s}$.

\section{Vertical impact, course of events}

Fig. 2 represents, schematically as true to scale as possible, the course of a vertical impact. The atmosphere which corresponds in mass only to a layer of rock of 3 to $4 \mathrm{~m}$ is penetrated without perceptible deceleration. The air shock, in spite of its very reasonable comparison to bomb blasts, is of no geological importance. In Fig. 2 a we start our time scale with the moment of contact between meteorite and ground.

Supposing similar rocky matter for both surfaces, they both will be compressed with one half of the

3 D. E. Gault and E. D. Heitowit, The Partition of Energy for Hypervelocity Impact Craters formed in Rock. Proc. 6th Hypervelocity Impact Symposium. meteorite speed of $22 \mathrm{~km} / \mathrm{s}$. According to Fig. 1 a particle velocity $u$ of $11 \mathrm{~km} / \mathrm{s}$ is connected with a shock velocity $U$ of $18.5 \mathrm{~km} / \mathrm{s}$. Fig. $2 \mathrm{~b}$ shows the state $0.032 \mathrm{~s}$ later. The shock wave, which travels nearly as a plane wave through the meteorite, has passed its lower half, while its upper half has moved on for $0.72 \mathrm{~km}$ quite undisturbed. The contact surface with its $11 \mathrm{~km} / \mathrm{s}$ has arrived at a depth of $0.36 \mathrm{~km}$. The shock in the ground has proceeded nearly $0.6 \mathrm{~km}$ downwards from the initial surface. This shock spreads like a spherical shock and decreases in amplitude accordingly.

In Fig. $2 \mathrm{c}$ a time interval of $0.064 \mathrm{~s}$ has elapsed. The shock running back has passed the whole meteorite and arrived at its highest point. The matter of meteorite and ground passed by the shock wave is now approximately in the following state:

pressure: 5 Mbar,

volume: 0.41 of the initial volume,

internal energy: $60 \mathrm{MJ} / \mathrm{kg}$,

thermal part thereof: $50 \mathrm{MJ} / \mathrm{kg}$,

velocity still: $11 \mathrm{~km} / \mathrm{s}$ downwards.

The internal energy is about 15 times that of high explosives and this energy is contained in $5000 \mathrm{Mton}$ of matter.

In this highly compressed state ionisation and dissociation are suppressed. Only some electronic excitation stores extra-energy. The specific heat will therefore not increase much. Between $1000^{\circ}$ and $2000^{\circ}$ it is $1.2 \mathrm{~kJ} /$ degree $\mathrm{kg}$. For $50 \mathrm{MJ} / \mathrm{kg}$ we should estimate the temperature to be about $30000^{\circ}$, or at least $20000^{\circ}$. It is much higher than the critical temperature of $\mathrm{SiO}_{2}$, which is to be expected near $5000^{\circ}$.

At the upper surface this highly compressed vapor borders on air of low density and expands explosively upwards. However, the whole internal energy, including the thermal one which can be reconverted into mechanical energy to perhaps $90 \%$ because of the high initial temperature, does not suffice for the matter to gain a relative velocity of $11 \mathrm{~km} / \mathrm{s}$ against the momentary state of downward movement. Relative velocity of $11 \mathrm{~km} / \mathrm{s}$ would mean an absolute velocity of zero. An upward movement in bulk cannot start at once. For this reason the upper border in Fig. $2 \mathrm{~d}$ has been drawn as indefinite, though in reality it will be sharp and lies somewhere.

4 E. Preuss, Das Ries und die Meteoritentheorie, Fortschr. Mineral. 41, 271 [1964]. 

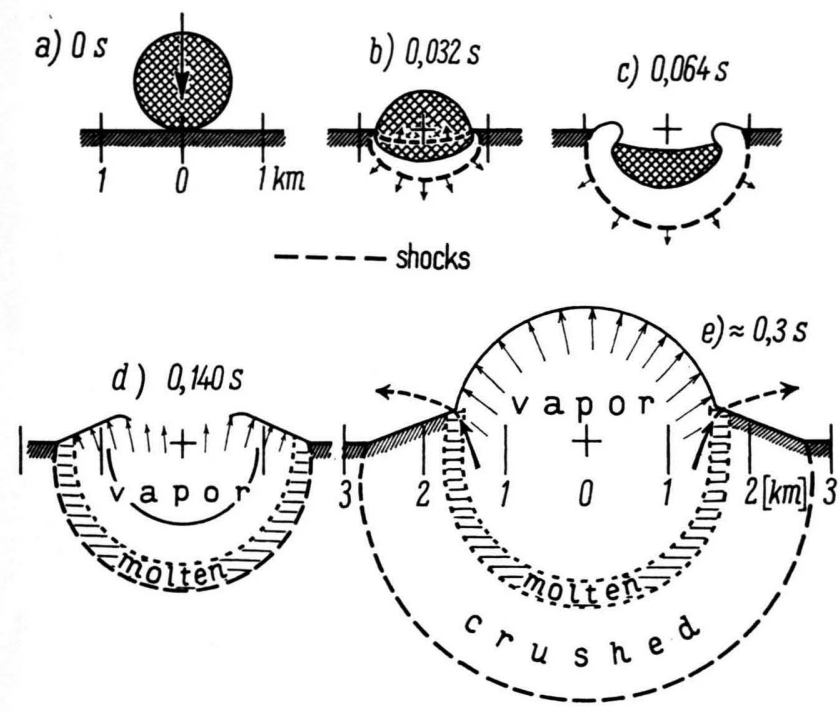

Fig. 2. Course of events for a vertical impact of a stony meteorite.

By means of elastic waves which pass the hot compressed vapour with a high speed in the order of 20 to $30 \mathrm{~km} / \mathrm{s}$ momentum is transported upwards from the bottom of the shock wave system and strong expansion begins. With a strong air shock before it the vapor first expands in a nearly half spherical form, as shown in Fig. $2 \mathrm{e}$.

For an instant we leave this expansion and regard the ground shock again. In gross approximation the particle velocity decreases as $r^{-3 / 2}$. From Fig. 1 we take the appropriate shock velocities and thermal energies. Up to a radius of $1.3 \mathrm{~km}$ the rock will be vapourized totally. After a zone of partial vapourisation the shell from 1.5 to $1.8 \mathrm{~km}$ will be molten. Further the rock will be crushed, at first rather finely, then coarser and coarser. At a distance of $5 \mathrm{~km}$ from the origin the cracking practically ceases because the shock pressure drops beneath 50 kbar. The fact that, especially at the Ries crater, the surface waves have cracked and thrown up the sedimentary layers to a greater distance, is of less interest for our problem now.

Where the shock wave passes along the surface, the ground begins to move upwards. The particle velocity of the shock has its essential component in the horizontal, directed away from the centre. Because the shock must be bent back towards the surface, there is also a vertical component of particle velocity. The relaxation of the pressure adds a vertical speed of the same amount as the horizontal

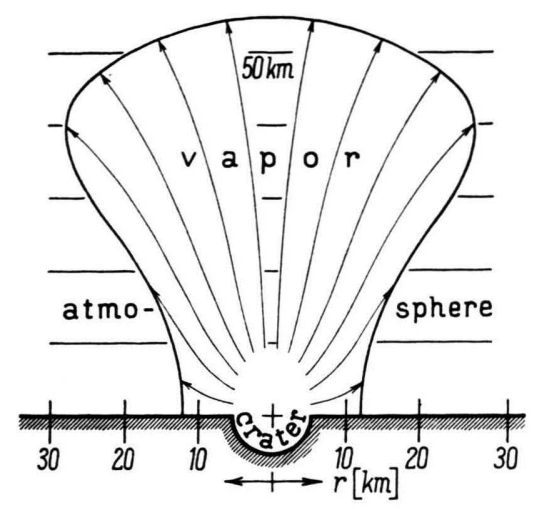

Fig. 3. Expansion flow of the rock vapour out of the atmosphere.

thereby causing the matter to ascend more steeply than $45^{\circ}$, up to $60^{\circ}$. The speed will begin with 3 or $4 \mathrm{~km} / \mathrm{s}$ and decrease with growing distance from the centre.

What ascends first near the rim of the impacted meteorite is vapour or partly vapourized rock. In Fig. $2 \mathrm{e}$ this vapour has not been distinguished from the hotter vapour in the central region. The first ascending matter which holds together in tough drops or patches is that which is molten to a sufficiently viscous state. Its path is indicated in Fig. $2 \mathrm{e}$ by the arrows. The property of being able to melt in viscous form is discriminating and may be most important for the selection of silica rich rock. Limestone for instance cannot be transformed into a glassy state.

The glassy patches ascend in a very dense cloud which spreads only slowly. This movement will soon be overrun by the very violent expansion of the vapour. It is of no importance, whether the time estimate of $0.3 \mathrm{~s}$ for this event is correct or whether this event occurs a little earlier or later. The path of the matter which could have formed tektites is forced to the horizontal along with the lower rim of the mighty vapour expansion flow. The dashed arrows in Fig. $2 \mathrm{e}$ indicate this path.

This flow will come to rest at a distance of 10 to $15 \mathrm{~km}$. The drops and patches transported with this flow fall down and mix up with the enormous quantities of debris of crushed and molten rock which leaves the crater later and more slowly.

The result is: A vertical impact under the conditions mentioned yields no tektites! The question is obvious: How must conditions be altered? 


\section{Expansion of the vapour}

The shocks have vapourized about $5 \mathrm{~km}^{3}$ of rock. $2 \mathrm{~km}^{3}$ of these are initially heated to a temperature of $20000^{\circ}$ to $30000^{\circ}$. The first adiabatic expansion which mainly serves to reverse the direction of movement, causes a rather rapid decrease in temperature. Further the still highly compressed vapour expands into the atmosphere. When by adiabatic cooling the boiling point is reached, the matter, of which $\mathrm{SiO}_{2}$ is the major part, begins to condense into a fine fog. The large latent heat of vapourisation of 4 to $5 \mathrm{MJ} / \mathrm{kg}$ set free by this process inhibits nearly quantitatively the further adiabatic drop of temperature. It can drop only as little as the boiling point decreases with decreasing pressure. In spite of the loss of vapour by condensation, this effect of preserving the temperature enhances the expansion ability of the vapour very much.

If we imagine the vapour of $5 \mathrm{~km}^{3}$ of rock at a pressure of 1 atmosphere and at the boiling point $2900^{\circ}$ of $\mathrm{SiO}_{2}$ and even admit condensation of one half of the matter to maintain the temperature, then a half-sphere with a radius of $23 \mathrm{~km}$ would be filled with this vapour. The density of this vapour including the weight of the condensed $\mathrm{SiO}_{2}$ fog would be $2 / 5$ of that of air.

These orders of magnitude clearly show: A halfspherical expansion is impossible. The vapour will expand in that direction, where the resistance of the atmosphere decreases exponentially, namely upwards. Qualitatively the picture of the expansion will look like Fig. 3. According to rough calculations the vapour expands with a velocity of 2 to $3 \mathrm{~km} / \mathrm{s}$. This expansion carries on to heights of several hundreds of $\mathrm{km}$ whereby finely condensed matter is distributed over large distances.

\section{Tektites}

If some matter suitable for tektites could be transported with this expansion flow, the problem would be solved. To heights of a least $50 \mathrm{~km}$ the viscous drops of glass move along with the vapour without being able to maintain any significant relative movement against the vapour flow. At heights of about $100 \mathrm{~km}$ the density of the vapour will gradually decrease to such low values that all aerodynamic forces practically cease to exist. Tektites continue their orbit in free flight till they dip again into the denser atmosphere. The expansion flow presumably contains relatively little turbulence. For this reason a small or rather sharply limited stray field of tektites is not controversial.

The tektites are protected from cooling by the ambient vapour. On the other hand, their temperature increase due to the influence of the somewhat hotter vapour during the short time of ascent is also unimportant. The condensation of vapour will not supply a thick layer because it happens in competition with the condensation on the much larger surface of the fine fog of condensed matter. It may be possible that for instance $\mathrm{SiO}_{2}$ condenses preferably.

Traces of gases and of water in the tektites will form bubbles, swelling with decreasing outer pressure, till they leave the tektite. Small remaining bubbles will be compressed to zero if the tektite is still viscous when subjected to pressure again. Atomic diffusion into or out of the interior of the tektite is impossible because of lack of time.

\section{Oblique impact and impact in pieces}

Oblique impact of a meteorite may offer a possibility to get tektites right into the expansion flow. What is the change in the course of the impact according to section 2 and Fig. 2?

We assume that the added horizontal component of impact velocity points to the right. Vertically everything remains unchanged, but there is scarcely a possibility of stopping the tremendously large horizontal momentum of the meteorite. The consequence is for Fig. $2 \mathrm{c}$ that the meteorite pushes itself to the right beneath the surface layers of the ground, augmenting the shock waves at the right side and attenuating them at the left. Penetration occurs to a little deeper extent than portrayed in Fig. 2 due to the somewhat larger density of a real meteorite over sedimentary and granitic rock, whereby pushing beneath the upper layers is favoured. Under such conditions in the phase corresponding to Fig. $2 \mathrm{e}$ the uppermost rim of shock wave molten rock may be pushed up along interior flow lines of the expansion flow that lead out of the atmosphere.

If this happens to some larger extent, it should stray out the tektites in form of a fan. Australia and Northern America seem to be examples for this effect.

We get a second possibility for tektite flight, if the meteorite breaks up into few large pieces within the 
earth's gravitational field. These pieces impact near to another and practically simultaneously. Just at the beginning each impact has its own vapour expansion. A moment later the individual expansion flows unite to one common flow. Tektitic matter which was caught right at the outermost rim of an individual flow may, by convergence with another flow, soon be on an interior flow line of the common flow.

At the Ries an impact of a fractured meteorite seems to be probable. Furthermore, it is a priori probable that the common impact direction of the pieces was oblique and not just vertical and that the pieces had an irregular form. Under these conditions tektitic matter may have entered the expansion flow anywhere. Due to a circular spreading of the flow, the tektites may be expelled by it in any direction, the forward direction being statistically favoured. However, this fact is quite meaningless for one single event.

Just as the direction of the moldavites will most likely have been defined by chance, so the other direction in which a border part of the expansion flow transported the Reuter blocks, will have been settled by another independent chance. The odd angle between both directions is no mystery.

An oblique impact of some irregular pieces must initially yield an irregular shock wave in the ground. Similar to the expansion flow in space, however, such a shock wave has a strong tendency for smoothing out all irregularities. Therefore the border of the crater and the distribution of the bulk of the throwout will tend to be circular.

This tendency renders it difficult to analyze the course of the details of the Ries event. On the other hand, the author does not at all want to assert that these difficulties inhibit the analysis. One fact is sure, the irregularities of the Ries offer us possibilities and probabilities for the flight of the moldavites as well as for the flight of the Reuter blocks.

Acknowledgements: The author thanks especially E. W. Adams, W. v. Engelhardt, W. Gentner, E. Preuss, W. Wimmenauer, and J. Zähringer for discussions, letters, and extra prints, and he thanks the lightgas-gun group of the French-German Research Institute for presenting the results of impact experiments. 